\title{
A NEW COMPACT MULTIBAND ANTENNA FOR STROKE DIAGNOSIS SYSTEM OVER 0.5-3 GHZ
}

\author{
Shirin Abtahi ${ }^{1}$, Jian Yang ${ }^{1}$ and Stefan Kidborg ${ }^{2}$
}

${ }^{1}$ Dept. of Signals and Systems, Chalmers University of Technology ${ }^{2}$ Medfield Diagnstics AB, Medicinargatan 8A, SE-41346 Gothenborg, Sweden

\section{Antenna characterization for Stroke Diagnosis Systems}

- Antenna size, particularly the size of its footprint, is very important in stroke diagnosis systems. Human brain is a very lossy material for microwave signal at high frequency. At a lower frequency, the signal penetration can be deeper, but the antenna size is larger. On the other hand, it is demanded to have more antenna elements in such the system for higher resolution. Therefore, the compactness of the antenna (small footprint) is critical for stroke diagnosis system due to the limitation of the area of human head.

- Reflection Coefficient $(\Gamma)$, at the antenna input port is a measure of how much the signal is transmitted by the antenna when it is located on the top of a human head model if the antenna has a low ohmic loss. Low ohmic-loss of the antenna is also emphasized.

- Radiation Leakage-out Ratio $R_{\text {leak }}$ is defined as the ratio of the power radiated out into free space $P_{\text {rad }}$ to the transmitted power $P_{\text {trans }}$

$$
R_{\text {leak }}=\frac{P_{\text {rad }}}{P_{\text {trans }}}
$$

If the antenna has a low ohmic loss, we have

$$
R_{\text {leak }} \approx \frac{P_{\text {rad }}}{(1-\Gamma) P_{\text {in }}}
$$

Where $P_{\text {in }}$ is the input power to the antenna.

The radiation leakage-out ratio measures the signal loss due to the signals radiated out into free space instead of being transmitted into human head. In addition, a high radiation leakage-out ratio will cause a strong interference among the elements in an antenna array used for diagnosis system. Therefore, a low radiation leakage-out ratio is one of the design goals.

The radiation leakage-out ratio is also an indirect measure of how directional the radiation characteristic of an antenna is in near-field sensing applications, such as stroke diagnosis and breast cancer detection, where the far-field radiation function fails to characterize this performance.

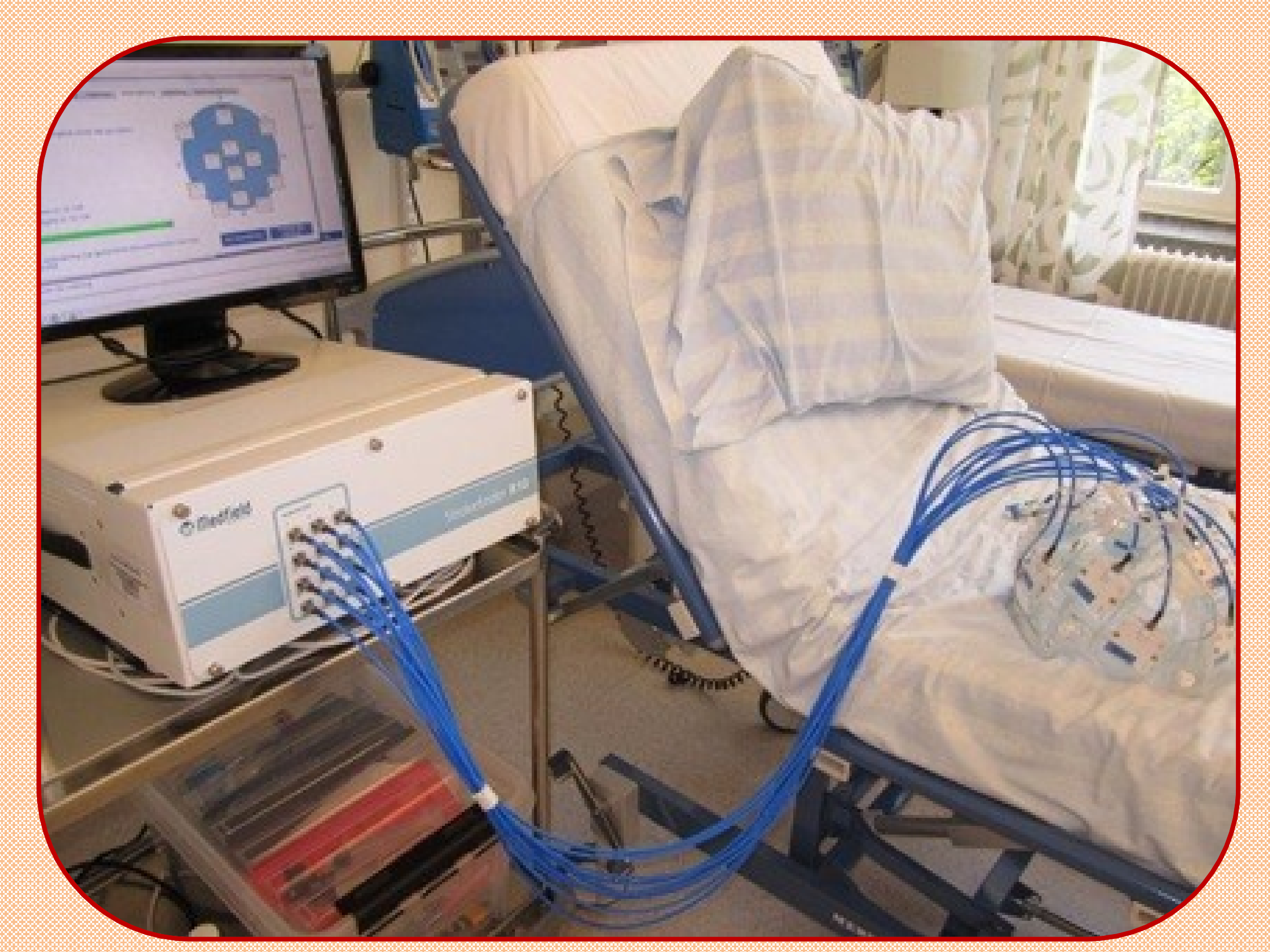

Existing stroke diagnosis system developed by Medfield Diagnostics (Stroke Finder)

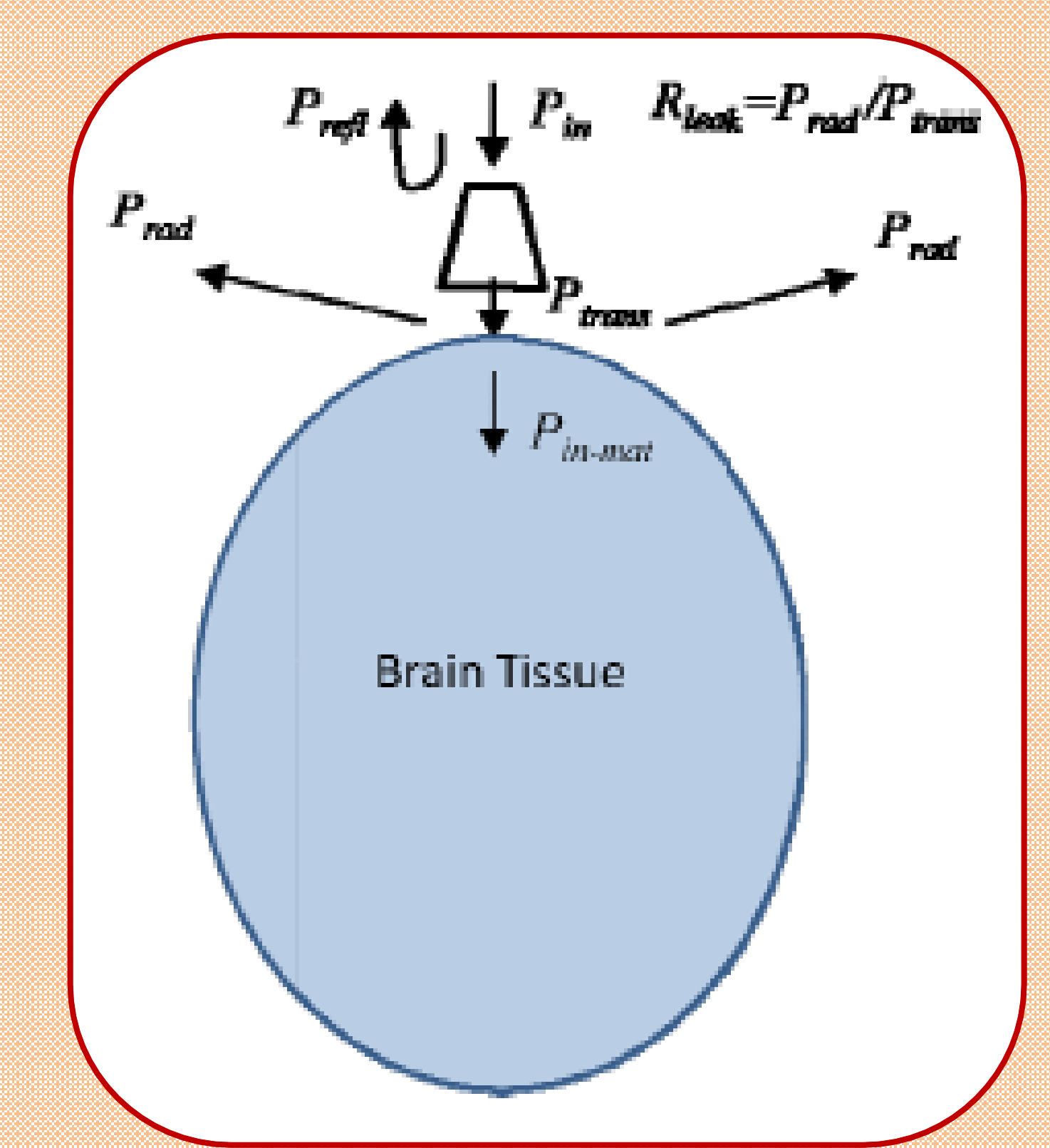

One antenna on the top of a hed in a stroke diagnosis system and definition of radiation leakage-out ratio

\section{Background}

The brain stroke is the third cause of death, ranking only behind heart disease and cancers. It also causes serious long-term disabilities which lead significant economic impact. Therefore, The real-time diagnosis is important because of different treatment: the ischemic stroke patients are given thrombolytic treatment which could be fatal for hemorrhagic patients. The microwave based techniques for stroke diagnosis need antennas in single or multi-frequency ranges. The multi-band operating systems are preferred due to higher resolution and deeper penetration than narrow band counterparts.

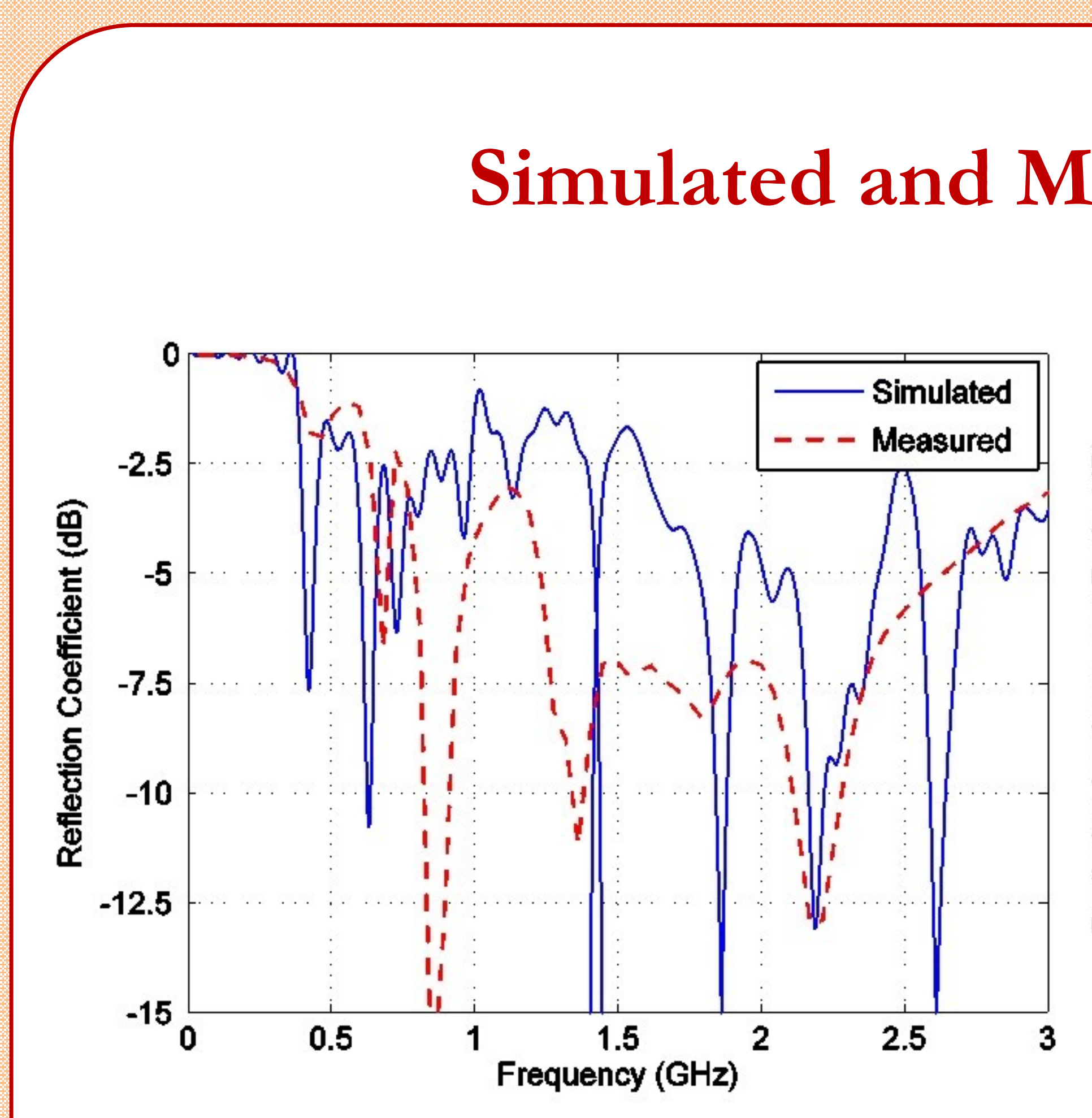

Simulated and measured reflection coefficients of the new antenna when it is located on top of the phantom head
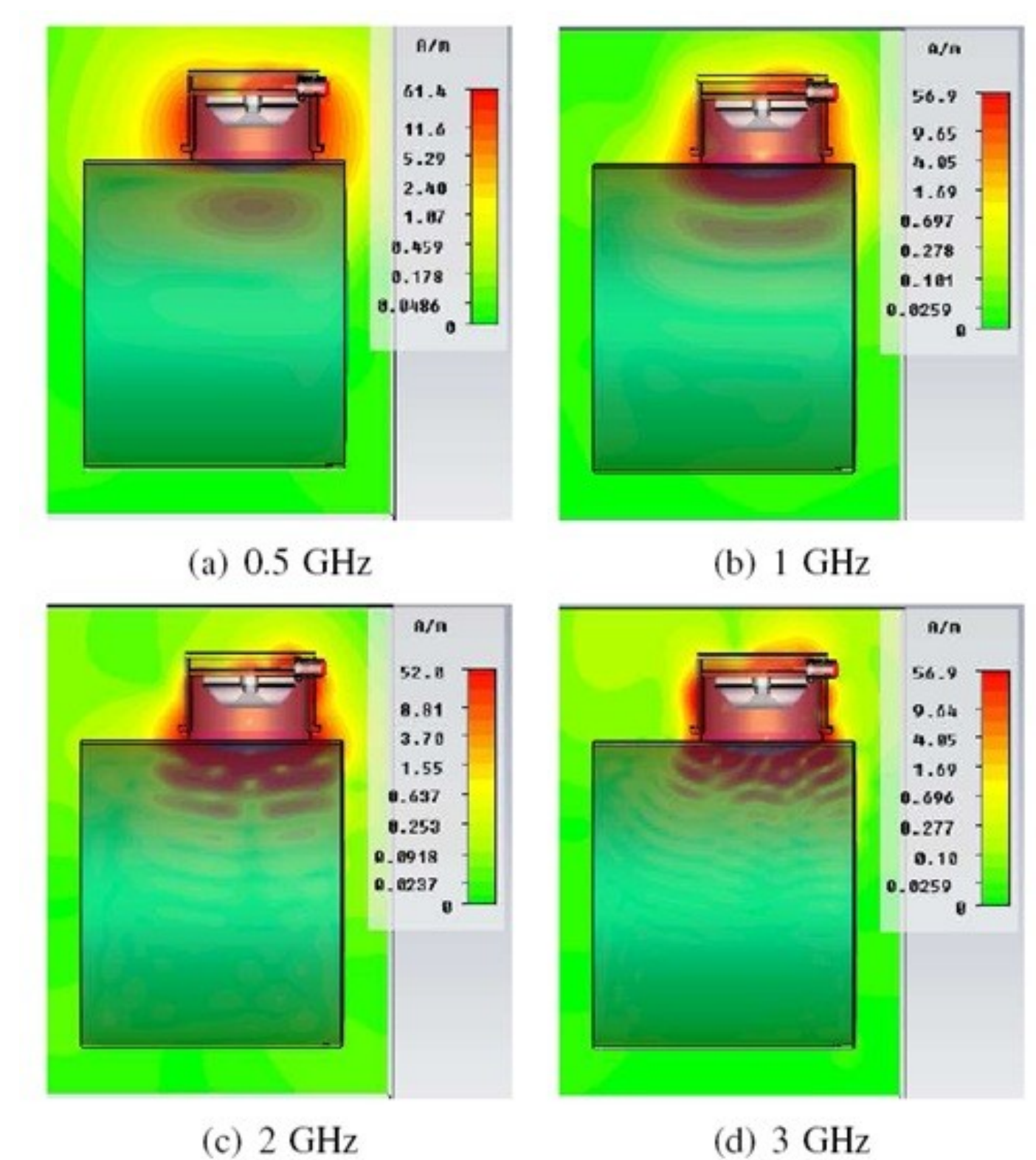

Simulated $\mathrm{H}$-field distribution inside the simple head model by using CST MWS

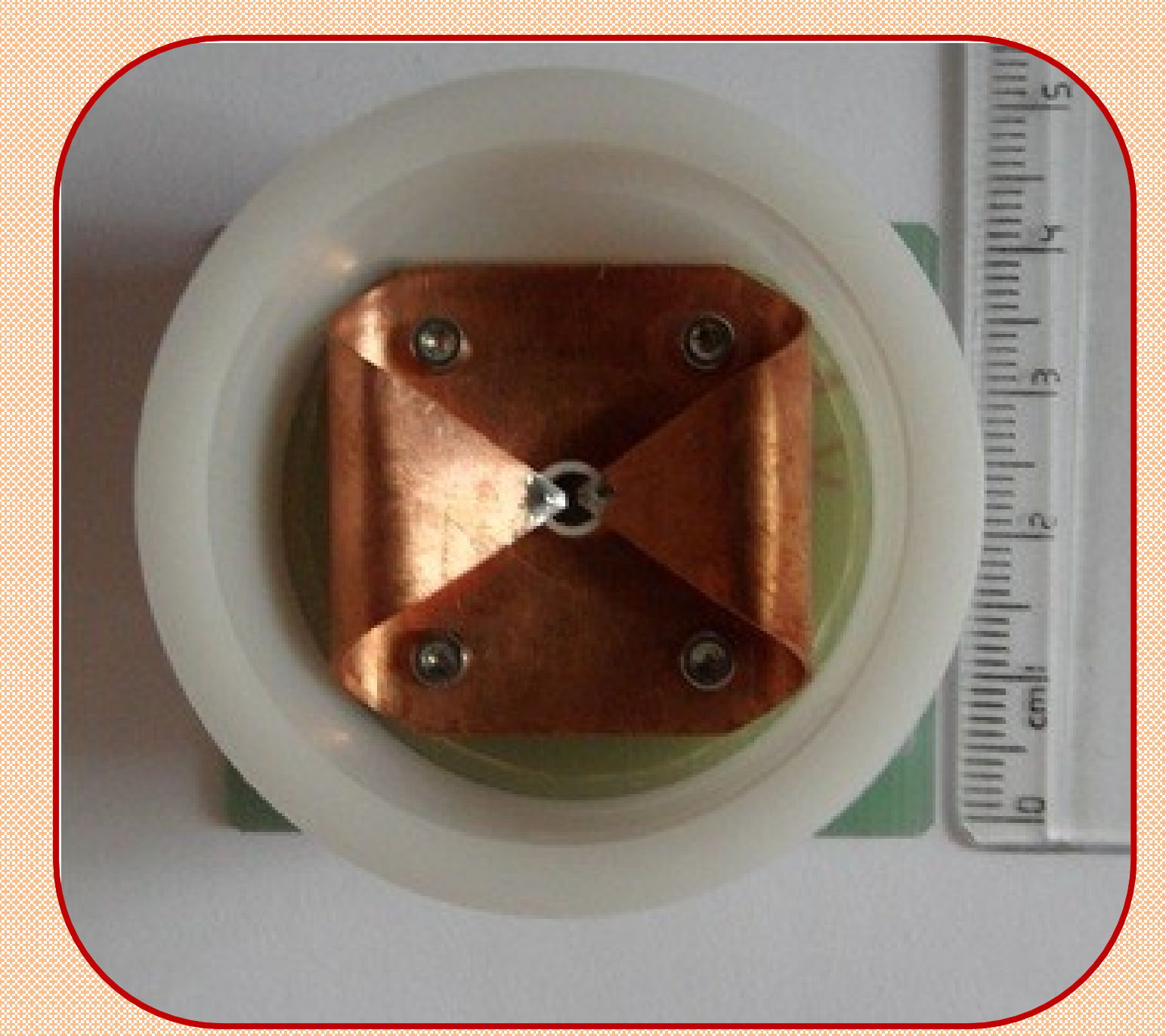

Manufactured Antenna

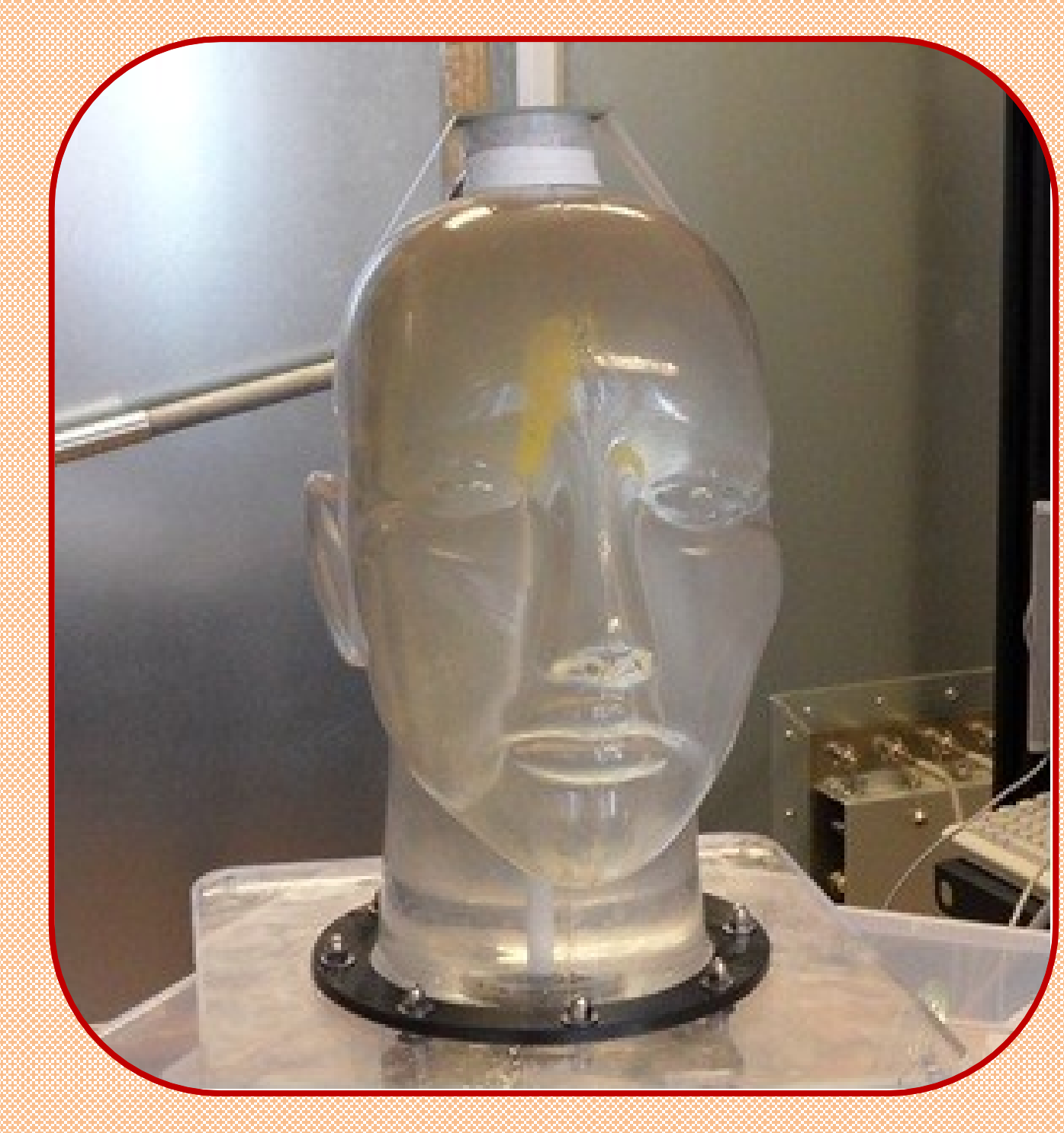

Phantom used for measurements

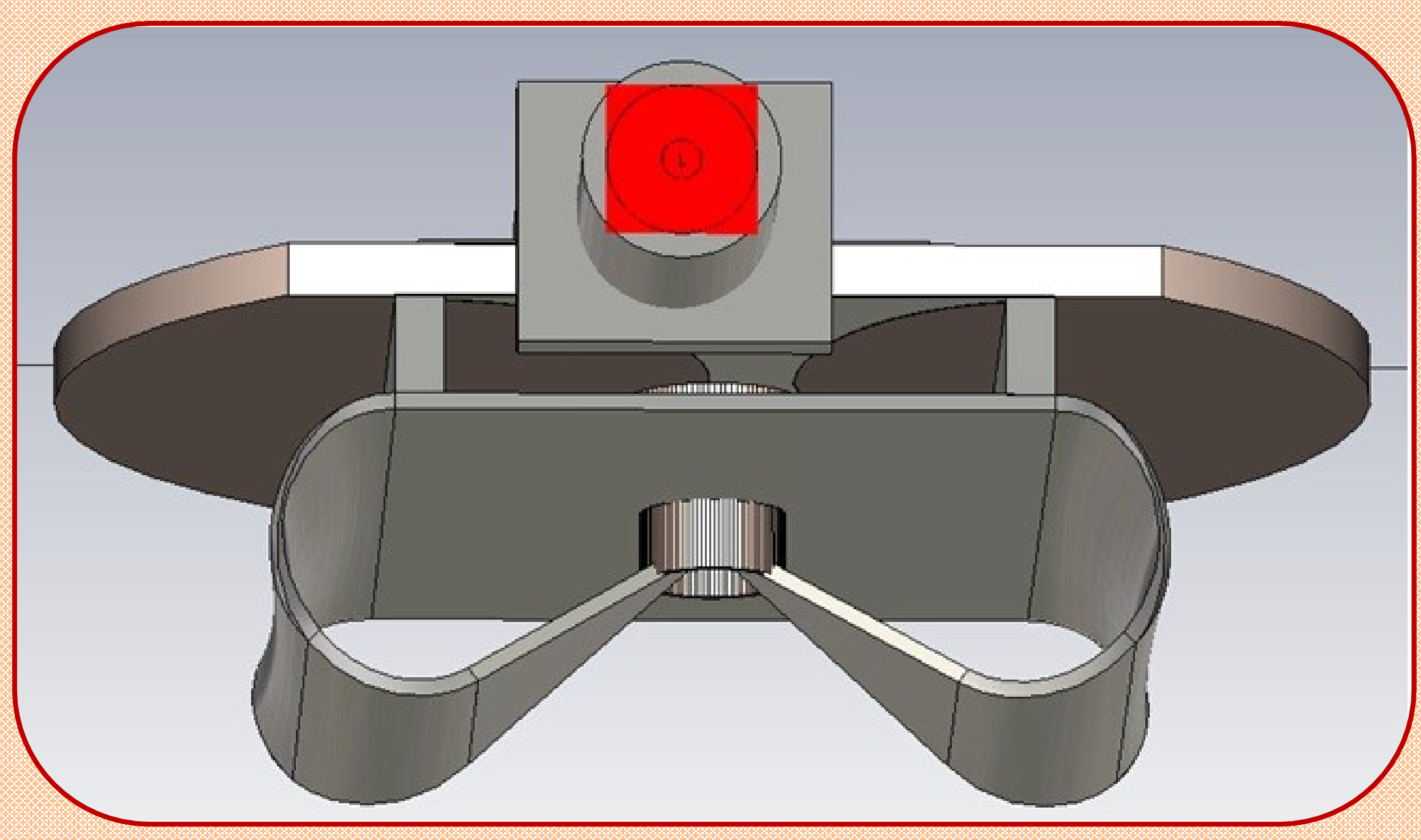

Simulated Antenna in CST MWS

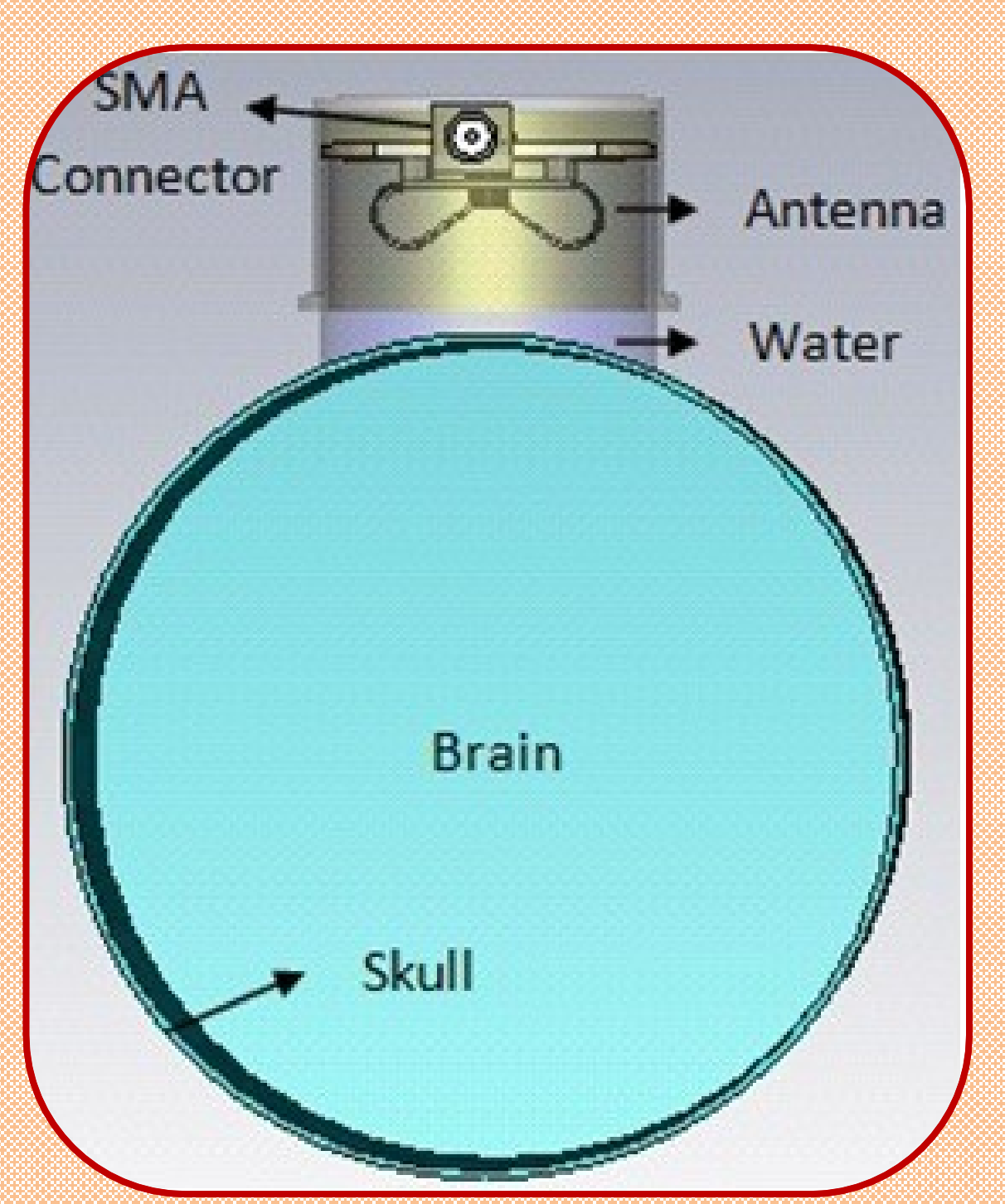

Simple Head Model used for simulations 